\title{
Hier sind Sie richtig!
}

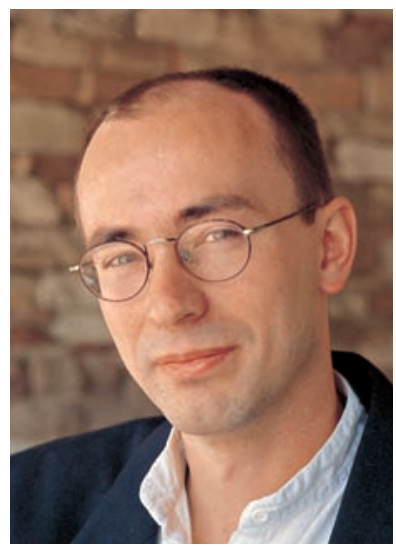

Dr. med. Dieter Schmid, Redaktionsleitung
Keine Angst! Sie sind hier richtig. Wenn Sie dieses Heft durchblättern, werden Sie sich vielleicht fragen, ob das wirklich Via medici ist, was Sie da in den Händen halten: Ich kann Sie beruhigen: Wir sind die Alten geblieben, aber pünktlich zum Jahresanfang haben wir Via medici ein neues Kleid geschneidert - mit frischen Farben, modernen Formen und optimierter Leserführung. Natürlich war das kein Schnellschuss aus der Hüfte. Wir haben uns mehrmals mit langjährigen Lesern beraten. Ein beruhigendes Ergebnis dieser Workshops: Inhaltlich treffen wir offensichtlich ins Schwarze. Ihre Kommiltonen baten uns, an unserer Themenpalette nur nicht zu viel zu ändern! Deshalb haben wir uns bei diesem „Relaunch“ vor allem auf das Layout konzentriert. Wir hoffen, dass Ihnen das Ergebnis zusagt. Gefällt Ihnen das neue Outfit von Via medici? Schreiben Sie uns an via.medici@ thieme.de. Jedes Feedback ist herzlich willkommen!

Ein weiteres Ergebnis unserer Workshops: Der Editorial-Schreiber soll sich kürzer fassen! Dem Wunsch komme ich natürlich nach ;-) Trotzdem möchte ich nicht versäumen, Ihnen zwei Artikel in dieser Via medici besonders ans Herz zu legen. Christian Fleischhauer berichtet in „Medizin beim islamischen Tiger“ auf S. 14 von seinem PJ-Wahl-Tertial in Malaysia. Er wurde dort hervorragend betreut und hat eine exzellente Ausbildung genossen. Gibt es trotz solcher Erfahrungen Argumente, die dafür sprechen, dass man Auslands-Tertiale beschränken sollte? Diese brisante Frage möchten wir in unserer aktuellen Online-Umfrage diskutieren (siehe unten). Außerdem sei Ihnen unser Artikel „Umgang mit Prüfungsmisserfolgen“ empfohlen (S. 18). Mit diesem Text wollen wir keine Panik schüren! Im Gegenteil: Wichtig ist, zu begreifen, dass eine Prüfung keine unüberwindbare Barriere ist, an der man „scheitern“ kann. Das Leben geht weiter - ob man nun einen zweiten Anlauf startet oder ob man den Misserfolg als Chance sieht, sich völlig neu zu orientieren. Wer diese „Message“ verinnerlicht, sieht Prüfungen bereits deutlich lockerer - und steigert damit seine Chancen, gar nicht erst durchzufallen!

Ich wünsche Ihnen ein wunderschönes, hoffnungsfrohes Neues Jahr 2008!

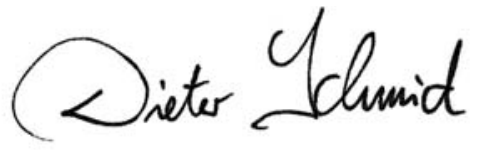

„Zum letzten Mal. Sie haben keinen Telefonjoker und Sie bekommen von mir auch keine vier Antwortalternativen. Sie sagen mir jetzt, wie dieser Nerv heißt, oder ..." 